\title{
Return cycle mapping: Have we come full cycle?
}

Yousuf Mahomed, MD

John M. Miller, MD

From the Division of Cardiovascular Surgery, Department of Surgery, Division of Cardiology, Department of Medicine, and Krannert Institute of Cardiology, Indiana University School of Medicine, Indianapolis, Ind.

Received for publication Sept 15, 2000; accepted for publication Oct 13, 2000.

Address for reprints: Yousuf Mahomed, MD, Division of Cardiovascular Surgery, Indiana University School of Medicine, 215 Emerson Hall, $545 \mathrm{~N}$ Barnhill Dr, Indianapolis, IN 46202.

J Thorac Cardiovasc Surg 2003;125:S17-9

Originally published in J Thorac Cardiovasc Surg 2001;121:197-9.

Copyright () 2003 by The American Association for Thoracic Surgery

0022-5223/2003 $\$ 30.00+0$

doi: $10.1067 / \mathrm{mtc} .2003 .213$

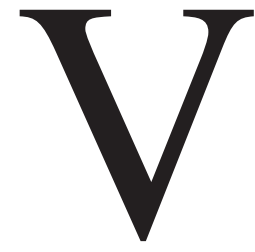

entricular tachycardia (VT) occurring in patients who have had a previous myocardial infarction has been demonstrated to be due to reentry. Critical components of this reentrant circuit-without which the tachycardia cannot continue- have been shown to be located within endocardial or subendocardial layers of the infarct zone or its periphery. This is fortunate, in that it allows a surgeon's knife or an electrophysiologist's catheter to have access to critical components of the VT circuit to incise, remove, or ablate this relatively small amount of tissue and thereby eliminate VT recurrences. In hearts that have already sustained muscle loss from infarction, it stands to reason that one should attempt to minimize the amount of additional damage done to eradicate the tissue responsible for VT. The process of electrophysiologic mapping has been a mainstay of surgical therapy for VT in that it attempts to localize these critical areas of the reentrant circuit, such that minimal incision, resection, or ablation could effect antiarrhythmic success. The more complete the mapping data, the better the arrhythmia cure rate. ${ }^{1}$ However, in practice, mapping has several limitations (some of which are discussed herein), and because one cannot always be certain that all arrhythmogenic areas have been identified, most surgeons use a generous resection or cryoablation margin beyond areas indicated by mapping-even to the point of removing all visible endocardial scar. $^{2}$

\section{Standard VT Mapping and Return Cycle Mapping}

During standard VT mapping, one attempts to locate areas of the endocardium from which diastolic potentials (timing between the end of one VT QRS and the next) are recorded. The central common pathway (CCP) of the VT circuit should be in such an area, although diseased tissue in other areas may masquerade as CCP by having similar diastolic timing. These areas of greatest interest are anatomically small (2-4 $\mathrm{cm}^{2}$ ), and recordings from them have very low amplitudes and great complexity, making their interpretation difficult. Nitta and associates ${ }^{3}$ have attempted to circumvent these problems with return cycle mapping. With this technique, one uses a short burst of overdrive pacing during VT ("entrainment") and records the timing of activation of large areas of endocardium during the first 2 beats of VT when it resumes after cessation of pacing. An isochronal line connecting recording sites at which the length of the return cycle equals the VT cycle length can be plotted. When pacing is performed at several different sites, a family of such isochrones is made; Nitta's group found that the point of intersection of these lines is at the CCP.

Is the return cycle mapping technique valid? It seems to reliably point to the entrance to the CCP. Nitta and colleagues ${ }^{3}$ have taken the necessary steps to validate the technique by terminating VT with cryoablation at sites to which they were directed by the return cycle mapping method. If the technique is so good, are there any limitations to its use? As it turns out, quite a few conditions have to be met before return cycle mapping can be applied successfully to yield its impressive results. Many of these apply to standard mapping as well.

1. Sustained uniform VT must be inducible during surgery. In at least $10 \%$ of cases, VT cannot be induced and its occurrence cannot be predicted before the procedure. Without sustained VT, return cycle mapping cannot be applied at all. 
2. VT must be entrainable; that is, overdrive pacing during VT must capture the heart and the same VT resume after pacing ceases. If VT terminates after pacing or changes to a different morphology, return cycle mapping cannot be used.

3. A large number of simultaneously recording electrodes must be in the heart, some of which must overlie the region of the $\mathrm{CCP}$, to form the necessary family of isochrones for return cycle mapping. This requires specialized equipment that is not available at all centers.

4. It must be possible to pace and capture at multiple sites, once again to form the set of isochrones whose intersection is at the CCP.

5. Finally, not all forms of reentry have a CCP, and the usefulness of return cycle mapping in such cases is unclear.

Return cycle mapping has not yet been evaluated in man; however, if the technique is validated in human VT mapping and ablation, it could prove useful as an additional tool. If a team of surgeons and electrophysiologists then plans to use return cycle mapping to cure a patient of VT, they can probably anticipate a good result if all the aforementioned conditions are met. However, they should also have contingency plans if any one condition fails. Alternate techniques include mapping areas of fragmented electrograms during sinus rhythm if VT cannot be initiated ${ }^{4}$ or using standard activation mapping techniques if overdrive pacing for return cycle mapping results in VT termination or change in VT morphology.

\section{The Big Picture}

Regardless of how useful return cycle mapping may be, a larger question remains: Is it needed? This is very much in doubt, because very few direct surgical procedures are performed currently. This is not for lack of good mapping techniques, but because alternatives exist. These include the implantable cardioverter-defibrillator (ICD) and catheter ablation for VT. In particular, the ICD has largely supplanted surgical ablation for therapy of VT by virtue of its superior peri-procedural morbidity and mortality, high efficacy and reliability, and ease of use. In cases of frequent VT episodes, catheter ablation (especially combined with ICD therapy) provides very good palliation or even cure. ${ }^{5,6}$ There are still circumstances in which neither of these therapies is reasonable and mapping-directed therapy for VT is preferable. 7,8 These include very frequent or incessant, hemodynamically poorly tolerated VT, known left ventricular thrombus (precluding catheter ablation), patient preference (potential for surgical cure rather than palliation), and the need for concomitant revascularization or valve surgery.

On this last point, most authorities believe that intraoperative VT mapping and ablation adds significant time and perhaps morbidity to the procedure. ${ }^{7,9,10}$ This concern raises the question of whether patients who have known risk factors for VT (prior infarction, especially with a left ventricular aneurysm) and are undergoing revascularization or valve surgery should have concomitant attempts at VT mapping and ablation or whether they should simply have an ICD inserted. In patients who have had preoperative spontaneous episodes of VT (and perhaps even syncope, which may have been caused by VT), concomitant arrhythmia surgery is usually warranted to prevent very frequent VT recurrences in the postoperative period. ${ }^{11}$ Because such cases are infrequent, once identified they should probably be referred to a center that maintains the required equipment and expertise to provide the best possible environment for surgery. In patients who have not yet had spontaneous arrhythmia episodes, the answer is less certain. Preoperative electrophysiologic study may clarify this, if it can be performed safely. ${ }^{12}$ Further complicating the decision is the fact that, for most surgical endocardial mapping and ablation techniques, an open ventricle is necessary-and, along with it, cardiopulmonary bypass. With increasingly frequent use of off-pump and minimally invasive revascularization techniques, adding an open-ventricle procedure negates the advantages of these techniques.

If return cycle mapping has missed its chance to make a major difference in surgical therapy of VT, perhaps it will have substantial application in endocardial catheter mapping of both ventricular and atrial macroreentrant arrhythmias (such as those occurring after atriotomy for septal defect or atrioventricular valve repair). Return cycle mapping could be used in conjunction with multipolar mapping systems such as noncontact arrays or basket contact catheters to localize the entrance to CCPs of these arrhythmias. ${ }^{13,14}$ To our knowledge, this technique has not yet been applied in these situations.

It is unlikely that return cycle mapping — or any mapping technique, for that matter-will ever replace the ICD for treatment of VT. ${ }^{8}$ If anything, ICD therapy is becoming more appealing because of the continued decrease in device size and increase in battery life, ease of implantation, reliability, and feature set. Surgery, on the other hand, continues to have a moderate operative morbidity and mortality and is not uniformly successful. In carefully selected cases, ${ }^{7,9}$ however, intraoperative mapping-perhaps with return cycle mapping - and surgical therapy can provide a very good outcome. Thus, although this new technique may not result in therapy for VT coming "full cycle" and returning to its origins in the operating room, it nonetheless offers new insights and options, which are always good to have.

\section{References}

1. Miller JM, Gottlieb CD, Marchlinski FE, Hargrove WC, Josephson ME. Does ventricular tachycardia mapping influence the success of antiarrhythmic surgery? [Abstract]. J Am Coll Cardiol. 1988;11:112A. 
2. Moran JM, Kehoe RF, Loeb JM, Lichtenthal PR, Sanders JH Jr, Michaelis LL. Extended endocardial resection for the treatment of ventricular tachycardia and ventricular fibrillation. Ann Thorac Surg. 1982;34:538-52.

3. Nitta T, Mitsuno M, Tokkas CK, Lee R, Schuessler RB, Boineau JP. Cryoablation of ventricular tachycardia guided by return cycle mapping after entrainment. J Thorac Cardiovasc Surg. 2000;121:249-58.

4. Bourke JP, Campbell RWF, Renzulli A, McComb JM, Cowan JC, Guzman F, et al. Surgery for ventricular tachyarrhythmias based on fragmentation mapping in sinus rhythm alone. Eur J Cardiothorac Surg. 1989;3:401-6.

5. Strickberger SA, Man KC, Daoud EG, Goyal R, Brinkman K, Hasse $\mathrm{C}$, et al. A prospective evaluation of catheter ablation of ventricular tachycardia as adjuvant therapy in patients with coronary artery disease and an implantable cardioverter-defibrillator. Circulation. 1997; 96:1525-31.

6. Rothman SA, Hsia HH, Cossú SF, Chmielewski IL, Buxton AE, Miller JM. Radiofrequency catheter ablation of postinfarction ventricular tachycardia: long-term success and the significance of inducible nonclinical arrhythmias. Circulation. 1997;96:3499-508.

7. Cox JL. Patient selection criteria and results of surgery for refractory ischemic ventricular tachycardia. Circulation. 1989;79:163-77.

8. Hargrove WC, Addonizio VP, Miller JM. Surgical therapy of ventric- ular tachyarrhythmias in patients with coronary artery disease. $J$ Cardiovasc Electrophysiol. 1996;7:469-80.

9. Nath S, Haines DE, Kron IL, Barber MJ, DiMarco JP. Regional wall motion analysis predicts survival and functional outcome after subendocardial resection in patients with prior anterior myocardial infarction. Circulation. 1993;88:70-6.

10. Page PL. Surgical treatment of ventricular tachycardia: indications and results. Arch Mal Coeur Vaiss. 1996;89:115-21.

11. Tam SK, Miller JM, Edmunds LH. Unexpected, sustained ventricular tachyarrhythmia after cardiac operations. J Thorac Cardiovasc Surg. 1991;102:883-9.

12. Kelly P, Ruskin JN, Vlahakes GJ, Buckley MJ Jr, Freeman CS, Garan $\mathrm{H}$. Surgical coronary revascularization in survivors of prehospital cardiac arrest: its effect on inducible ventricular arrhythmias and long-term survival. J Am Coll Cardiol. 1990;15:267-73.

13. Eldar M, Ohad DG, Goldberger JJ, Rotstein Z, Hsu S, Swanson DK, et al. Transcutaneous multielectrode basket catheter for endocardial mapping and ablation of ventricular tachycardia in the pig. Circulation. 1997;96:2430-7.

14. Schilling R, Peters N, Jackman WM, Davies DW. Mapping and ablation of ventricular tachycardia using a novel non-contact mapping system. Pacing Clin Electrophysiol. 1997;20:1089. 